\title{
Effects of La, Gd, or Lu Co-doping on Crystal Growth and Scintillation Properties of
}

Eu:SrI ${ }_{2}$ Single Crystals

Kei Nishimoto ${ }^{1}$, Yuui Yokota ${ }^{2}$, Shunsuke Kurosawa ${ }^{1,2}$, Jan Pejchal ${ }^{1}$, Kei Kamada ${ }^{2}$, Valery Chani ${ }^{1}$, Akira Yoshikawa $^{1,2}$

1. Institute for Materials Research, Tohoku University

2-1-1, Katahira, Aoba-ku, Sendai, Miyagi, 980-8577, JAPAN

2. New Industry Creation Hatchery Center (NICHe), Tohoku University

6-6-10, Aoba, Aramaki, Aoba-ku, Sendai, Miyagi, 980-8579, JAPAN 


\section{Introduction}

Scintillating single crystals have been widely used in various applications including medical imaging, homeland security, gamma-ray astronomy, resource exploration, and other fields [1-5]. Common requirements for the scintillators produced for such applications are high scintillation light output and energy resolution. These properties are necessary to achieve high spatial resolution of obtained images, high sensitivity to radiation. The scintillating crystals of halides, including chlorides, bromides, and iodides, have been widely studied due to their relatively high light yield and energy resolution originating from their narrow band-gap [6]. Recently, $\mathrm{Eu}: \mathrm{SrI}_{2}$ scintillating crystals (orthorhombic, Pbca group[7]) have attracted worldwide attention because they had demonstrated extremely high light yield and approximately 3\% energy resolution [8,9]. However, the Eu: $\mathrm{SrI}_{2}$ crystals are extremely hygroscopic. Therefore, growth of these crystals of acceptable optical quality is complicated task. Up to now, these crystals are mostly produced by Vertical Bridgeman (VB) method in a quartz ampoule that are well isolated from environmental atmosphere. However, the growth rate in the VB method is not sufficiently high. On the other hand, the micro-pulling-down ( $\mu$-PD) technique [10] is known to be very efficient when fast fabrication of oxide or halide crystals is desired. Therefore, we had modified the $\mu$-PD method to make it appropriate for the crystal growth of halide materials. The newly developed technology ensured complete isolation of highly hydroscopic starting materials and products (crystals) from environmental atmosphere containing oxygen and moisture [11]. It is noted that basic scintillation properties of Eu: $\mathrm{SrI}_{2}$ crystals grown by the modified $\mu$-PD method were reported recently [12].

Eu: $\mathrm{SrI}_{2}$ scintillator crystals have been widely investigated due to the high light yield and energy resolution as represented by divalent cation substitution [13] and halide mixed compounds [14]. However, the light yield and energy resolution values reported in the past [9] varied significantly that was also confirmed by our recent studies on the Eu:SrI ${ }_{2}$ crystals grown by the modified $\mu$-PD method. Our results indicated intermediate light yield values when compared to those reported by other groups. In one of the previous reports, the defect-mediated emission originated from some forms of oxidation or hydration in $\mathrm{SrI}_{2}$ ceramics was observed [15]. Those results leaded to conclusion that the instability of the light yield is attributable to the defects or impurities present in the crystal and assigned to $\mathrm{O}^{2-}$ anion. Especially, iodide crystals can easily accommodate $\mathrm{O}^{2-}$ anions at the $\mathrm{I}^{-}$sites of the crystals when there are some sources of $\mathrm{O}^{2-}$ anions around the crystals during their growth. If the Eu: $\mathrm{SrI}_{2}$ crystal contains $\mathrm{O}^{2-}$ impurity at the $\mathrm{I}^{-}$site, then iodine vacancy is generated or charge of the $\mathrm{Eu}^{2+}$ cation is changed to the $\mathrm{Eu}^{3+}$ according to the electroneutrality principle. The formations of the $\mathrm{I}^{-}$vacancies and/or $\mathrm{Eu}^{3+}$ cation are supposed to decrease the light yield.

In this paper, growth of Eu: $\mathrm{SrI}_{2}$ single crystals doped by trivalent $\mathrm{RE}^{3+}$ cations of $\mathrm{La}^{3+}, \mathrm{Gd}^{3+}$, or $\mathrm{Lu}^{3+}$ is discussed. It was assumed that these dopants incorporated into $\mathrm{Sr}^{2+}$ sites, and this way charge compensation of $\mathrm{O}^{2-}$ impurity was realized. If $\mathrm{RE}$ co-doping ions can occupy $\mathrm{Sr}^{2+}$ sites, then they can probably suppress generation of the $\mathrm{I}^{-}$vacancies or $\mathrm{Eu}^{3+}$ ions initiated by $\mathrm{O}^{2-}$ impurity in the crystal grown without RE co-doping. In addition, the scintillation properties of the RE co-doped Eu:SrI ${ }_{2}$ crystals were examined and compared with those produced without RE co-doping.

\section{Experimental}

Starting materials of nominal compositions of $\left(\mathrm{Sr}_{1-x-y} \mathrm{Eu}_{x} A_{y}\right) \mathrm{I}_{2}[x=0.075, y=0,0.01, A=\mathrm{La}$, Gd, and Lu] were prepared from high-purity powders of $\mathrm{SrI}_{2}$ (Alfa Aesar, 4N), $\mathrm{LaI}_{3}$ (Aldrich, 3N), EuI 2 (Aldrich, 3N), $\mathrm{GdI}_{3}\left(\right.$ Aldrich, $4 \mathrm{~N}$ ) and $\mathrm{LuI}_{3}(\mathrm{Aldrich}, 3 \mathrm{~N}$ ) in a glove box that was filled with Ar gas. The mixtures were then charged into a carbon crucible equipped with a $\phi 2 \mathrm{~mm}$ outlet orifice at the center of the bottom. Thereafter, the hot zone was assembled from the crucible, aluminum insulators, and quartz cover, and all this construction was placed into removable chamber. After that, the chamber was isolated from surrounded atmosphere and removed from the glove box with the closed gate valve. The chamber was then fixed to the growth system and connected with a turbo molecular pump. Finally, the chamber was vacuumed up to $10^{-4} \mathrm{~Pa}$ for several hours. During this vacuuming process, the crucible was heated to $\sim 300^{\circ} \mathrm{C}$ by the high-frequency induction coil in order to remove the moisture remain on the surfaces of starting materials, the crucible, the insulators, and quartz tubes. After the baking process, high purity Ar gas (99.9999\%) was injected and the crucible was heated up to the melting point of Eu:SrI ${ }_{2}$. The crystal growth was performed by pulling-down the melt using a Pt wire as a seed, and the pulling-down rate was in the range of $0.05 \sim 0.1 \mathrm{~mm} / \mathrm{min}$. The as grown crystals were cut and polished in mineral oil to produce specimens suitable for the measurements of optical and scintillation properties. As for the La co-doped Eu:SrI $2,100 \%$ of the melt was solidified into the single crystal. This crystal was cut to five pieces of the same length to analyze effect of solidification fraction on physical performance of the material.

The phase identification and the lattice parameter measurements were performed by the powder X-Ray Diffraction (XRD) method (RIGAKU, RINT2000) using isolated chamber that was equipped with a beryllium window. Radioluminescence spectra were examined with the Fixed Imaging Compact Spectrographs (Thermo Oriel Instruments \#77441) and Charge Coupled Device camera (Andor DU420-OE) using X-ray as an excitation source. These measurements were also performed in a hermetically-sealed chamber with a beryllium window. Actual concentrations of the cations in the crystals were evaluated by the inductively Coupled Plasma (ICP) analysis (Thermo Fisher Scientific, IRIS Advantage DUO). Pulse-height spectra of the polished crystals under $\gamma$-ray irradiation from

${ }^{137} \mathrm{Cs}$ radiation source were also inspected to estimate their light yields and energy resolutions. The non-proportionality was measured using $\gamma$-ray irradiation from ${ }^{137} \mathrm{Cs},{ }^{22} \mathrm{Na},{ }^{57} \mathrm{Co},{ }^{133} \mathrm{Ba}$, and ${ }^{241} \mathrm{Am}$. The polished crystals were optically coupled with a Photomultiplier Tube (PMT) (HAMAMASTU, R7600U) by optical grease in the glove box. Signals from the PMT were converted to digital data by a multi-channel analyzer (AMPTEK CO. Pocket MCA 8000A). At the same time, their decay curves were also measured using an oscilloscope (TEKTRONIX, TBS1102).

\section{Results and discussion}

Fig. 1 shows the liquid-solid interface observed by the CCD camera at stages of seeding (Fig.1a) and stationary growth Fig.1b). After complete melting of the starting mixture, the seed made of twisted Pt wires was brought into physical contact with the meniscus. Thereafter, the melt was pulled out of the interior part of the crucible by pulling-down the seed. Pulling rate was 
approximately $0.1 \mathrm{~mm} / \mathrm{min}$, and the liquid-solid interface below the bottom of the crucible was stable during entire growth process. The as-grown Eu7.5\%: $\mathrm{SrI}_{2}$ and $\mathrm{La}$, Gd or Lu co-doped Eu7.5\%: $\mathrm{SrI}_{2}$ single crystals are illustrated in Fig.2(a). All the crystals were approximately $\phi 2 \mathrm{~mm}$ in diameter and several centimeters in length. No cracks or inclusions were detected in the polished crystals under visual and microscopic observations. The results chemical compositions analysis performed by the ICP method indicated that the actual concentrations of $\mathrm{Sr}, \mathrm{Eu}$ and $\mathrm{La}$ in the crystals with nominal (melt) compositions of Eu7.5\%:SrI $2, \mathrm{Eu}^{2} .5 \%, \mathrm{La}_{1} \%: \mathrm{SrI}_{2}$, $\mathrm{Eu} 7.5 \%, \mathrm{Gd}_{1 \%}: \mathrm{SrI}_{2}$ and Eu7.5\%,Lu1\%:SrI 2 were $\mathrm{Sr}: \mathrm{Eu}=92.6: 7.4, \mathrm{Sr}: \mathrm{Eu}: \mathrm{La}=92.57: 7.12: 0.31, \mathrm{Sr}: \mathrm{Eu}: \mathrm{Gd}=92.22: 7.77: 0.01$ and $\mathrm{Sr}: \mathrm{Eu}: \mathrm{Lu}=92.09: 7.90: 0.01$, respectively. The difference between compositions of the melts and those of the corresponding crystals was result of segregation phenomenon. The segregation coefficient of $\mathrm{La}^{3+}$ (Ionic radius: $1.06 \AA$ ) with the ionic radius closest to that of the $\operatorname{Sr}^{2+}(1.16 \AA)$ host cation was relatively high. However, that of the $\mathrm{Gd}^{3+}(0.938 \AA)$ and $\mathrm{Lu}^{3+}(0.848 \AA)$ ions was considerably low [16].

The above ICP results demonstrated that segregation coefficients of all co-doping RE including La, Gd or Lu were less than unity. That is why, non-homogeneous distribution of all these co-dopants along the growth axis was expected. From industrial point of view, such non-uniform crystals are mostly useless. However, such materials are often used for research purposes to understand effect of dopant content on physical properties. Regarding La co-doped Eu7.5\%: $\mathrm{SrI}_{2}$ single crystal, this material was used to examine dependence of scintillation properties on dopant concentration (Fig.2(b)). The as grown crystal was cut into five equal parts along the growth direction, and every fragment was polished to produce specimens acceptable for the measurements of scintillation properties. In addition, their actual chemical compositions were evaluated by the ICP analysis after the measurements. These five specimens were identified as samples $1,2,3,4$, and 5 in order. Thus, the sample 1 corresponded to beginning of growth (solidification fraction of $0-20 \%$ ) and sample 5 corresponded to finalizing of the growth (solidification fraction of $80-100 \%$ ). The as polished specimens had thicknesses of approximately $0.7 \mathrm{~mm}$ and indicated high transparency. The cation ratios of the sample 1, 2, 3, 4, and $5 \mathrm{mere} \mathrm{Sr}:$ Eu : La $=91.58: 8.41: 0.01,91.61: 8.38: 0.01,91.67: 8.32: 0.01,91.73: 8.25: 0.02$ and $91.66: 8.30: 0.04$, respectively. The actual Eu concentrations in all the specimens were almost the same as the nominal (melt) compositions. Therefore, no inhomogeneity of Eu distribution along to the growth direction was detected. This is result of high similarity of ionic radii of $\mathrm{Eu}^{2+}$ and $\mathrm{Sr}^{2+}$ ions [16]. In contrast, actual La concentrations in these specimens were smaller than that in the melt (nominal composition) due to the mentioned non-uniform distribution along the growth direction.

The results of powder XRD analysis for the $\mathrm{La}, \mathrm{Gd}$ and $\mathrm{Lu} 1 \%$ co-doped Eu7.5\%: $\mathrm{SrI}_{2}$ crystals are illustrated in Fig.3. All the diffraction peaks of these XRD patterns were identified as those corresponding to the $\mathrm{SrI}_{2}$ structure, and no secondary phases were detected. The peaks intensities for each specimen were different most probably because the powders for XRD measurements were prepared from the grown crystals and there was a possibility that the powders contained some particles with particular facet plane. Lattice parameters of the crystals were calculated from the powder XRD patterns with Ti powder as the internal standard. The lattice parameters of all the grown crystals were almost constant regardless of Eu concentration and RE co-dopant content. Clear effect of co-doping on the lattice parameter was not observed due to the low concentrations of co-doping RE ions in the crystals.

Radioluminescence spectra of the polished La 1\% co-doped Eu7.5\%: $\mathrm{SrI}_{2}$ crystals were investigated under X-ray irradiation, and the results are demonstrated in Fig.4. Emission peak originating from $\mathrm{Eu}^{2+} 5 \mathrm{~d}-4 \mathrm{f}$ transition was observed around wavelength of 435 $\mathrm{nm}$ for all the crystals and the wavelength of the emission peak was almost the same as in previous reports [7]. The effect of La co-doping on wavelength of emission peak was not observed. In addition, emission peak from Eu ${ }^{3+}$ ion was not observed in the $\mathrm{Eu} 7.5 \%: \mathrm{SrI}_{2}$ and La co-doped Eu7.5\%: $\mathrm{SrI}_{2}$ crystals.

The light yields of the Eu7.5\%: $\mathrm{SrI}_{2}$ and La co-doped Eu7.5: $\mathrm{SrI}_{2}$ specimens were evaluated from the pulse-height spectra examined under $\gamma$-ray irradiation from ${ }^{137} \mathrm{Cs}$ using PMT in the glove box. Fig. 5(a) shows the pulse-height spectra with clearly resolved photo-peaks. The photo-peaks were fitted by the Gaussian function to obtain their positions and calculate the energy resolutions. The light yields were estimated by comparing the photo-peak positions between La co-doped Eu:SrI ${ }_{2}$ specimens and BGO standard crystal. Fig. 5(b) is the La concentration dependence of the light yield and energy resolution. The light yields of all La co-doped Eu7.5\% $\mathrm{SrI}_{2}$ specimens were lower than that of the $\mathrm{Eu} 7.5 \%: \mathrm{SrI}_{2}$ specimen.

Fig. 6 shows the non-proportionality of Eu7.5\%: $\mathrm{SrI}_{2}$ and La co-doped Eu7.5\% $\mathrm{SrI}_{2}$ specimens observed under $\gamma$-ray irradiation from ${ }^{137} \mathrm{Cs},{ }^{22} \mathrm{Na},{ }^{57} \mathrm{Co},{ }^{133} \mathrm{Ba}$ and ${ }^{241} \mathrm{Am}$ radiation sources. The non-proportionality of Eu7.5\%:SrI 2 was decreased with La co-doping. The results of light yield and non-proportionality suggest that the decrease of the light yield and the degradation of non-proportionality with La co-doping is attributable to the increase of concentration of some defects originated from La co-doping. However, nature of these defects is not clear now.

The scintillation decay curves of Eu7.5\%: $\mathrm{SrI}_{2}$ and La co-doped Eu7.5\% $\mathrm{SrI}_{2}$ specimens under $\gamma$-ray irradiation are illustrated in Fig. 7. All the decay curves were fitted by a single exponential decay equation. The obtained decay times for all the specimens were almost consistent with the previous report approaching the value of $1.1 \mu \mathrm{sec}$. Therefore, no evident effect of La co-doping on the decay time was observed.

\section{Conclusion}

Single crystals of $\mathrm{La}, \mathrm{Gd}$ or Lu co-doped $\mathrm{Eu}: \mathrm{SrI}_{2}$ were grown by the modified $\mu$-PD method. Possible effects of La co-doping on scintillation properties of the materials were examined. The results of powder XRD measurements indicated that all the crystal were single phase materials with $\mathrm{SrI}_{2}$ structure. Actual concentrations of $\mathrm{La}^{3+}, \mathrm{Gd}^{3+}$ and $\mathrm{Lu}^{3+}$ ions in the crystals were lower than those in the melt (nominal composition), and the segregation coefficient of $\mathrm{La}^{3+}$ in this crystal was less than unity. Oppositely, actual Eu ${ }^{2+}$ ion concentrations were equivalent to those in the melts. The light yield of Eu: $\mathrm{SrI}_{2}$ crystal decreased with increase of La co-doping. The decay times of La 1\% co-doped Eu7.5\%: $\mathrm{SrI}_{2}$ specimens were approximately $1.1 \mu$ sec. $\quad$ It is assumed that La co-doping generates some defects in the crystals that may trap charge carriers. Therefore the decrease of the light yield is observed. 


\section{Acknowledgment}

This work was partially supported by Japan Society for the Promotion of Science (JSPS) Research Fellowships for Young Scientists and Grant-in-Aid for Exploratory Research (25600130). In addition, this word was supported by Japan Science and Technology Agency (JST) for Development of Systems and Technology for Advanced Measurement and Analysis. We would like to thank Analytical Research Core for Advanced Materials, Institute for Materials Research, Tohoku University for their support. 


\section{Reference}

[1] I. Valais, S. David, C. Michail, D. Nikolopoulos, P. Liaparinos, D. Cavouras, I. Kandarakis, G.S. Panayiotakis, Nuclear Instruments and Methods in Physics Research Section A: Accelerators, Spectrometers, Detectors and Associated Equipment, 580 (2007) 616-614.

[2] A. Olivo, K. Ignatyev, P.R.T. Munro, R.D. Speller, Nuclear Instruments and Methods in Physics Research Section A: Accelerators, Spectrometers, Detectors and Associated Equipment, 610 (2009) 601-614.

[3] G.L. Case, M.L. Cherry, J.G. Stacy, Nuclear Instruments and Methods in Physics Research Section A: Accelerators, Spectrometers, Detectors and Associated Equipment, 563 (2006) 355-358.

[4] P. Chiozzi, V. Pasquale, M. Verdoya. Journal of Geochemical Exploration, 93 (2007) 13-20.

[5] Conny C.T. Hansson, Alan Owens, Johannes v.d. Biezen, Acta Astronautica, 93 (2014) 121-128.

[6] V.A. Pustovarov, I.N. Ogorodnikov, A.A. Goloshumova, L.I. Isaenko, A.P. Yelisseyev, Optical Materials, 34 (2012) $926-930$.

[7] H. Bärnighausen, E. Th. Rietschel, Z. allgemeine Chemistry, 354 (1967) 23-26.

[8] N. J. Cherepy, G. Hull, A. D. Drobshoff, S. A. Payne, E. van Loef, C. M.Wilson, K. S. Shah, U. N. Roy, A. Burger, L.A. Boatner,W.-S. Choong, and W. W. Moses, Applied Physics Letters 92(2008) 083508 1-083508 3.

[9] Edgar V. van Loef, Cody M. Wilson, Nerine J. Cherepy, Giulia Hull, Stephen A. Payne, Woon-Seng Choong, William W. Moses, Kanai S. Shah. IEEE Transactions on Nuclear Science 56 (2009) 3.

[10] H. Ogino, A. Yoshikawa, M. Nik1, A. Krasnikov, K. Kamada, T. Fukuda, Journal of Crystal Growth 287 (2005) 335.

[11] Y. Yokota, N. Kawaguchia, K. Fukuda, T. Yanagida, A. Yoshikawa, M. Nik1, Journal of Crystal Growth 318 (2011) 908.

[12] Y.Yokota, K.Nishimoto, ShunsukeKurosawa, DaisukeTotsuka, AkiraYoshikawa, Journal of Crystal Growth 375 (2013) $49-52$.

[13] J. S. Neal, L. A. Boatner, J. O. Ramey, D. Wisniewski, J. A. Nuclear Instruments and Methods in Physics Research Section A: Accelerators, Spectrometers, Detectors and Associated Equipment, 643 (2011) 75-78.

[14] E.D.Bourret-Courchesnen, G.A.Bizarri, R.Borade, G.Gundiah, E.C.Samulon, Z.Yan, S.E.Derenzo. Journal of Crystal Growth 352 (2012) 78-83.

[15] Stephen R. Podowitz, Romain M. Gaumé, Wesley T. Hong, Atlal Laouar, and Robert S. Feigelson. IEEE TRANSACTIONS ON NUCLEAR SCIENCE, 57 (2010) 6

[16] Y.Q.Jia. journal of Solid State Chemistry, 95(1991)184 
(a)

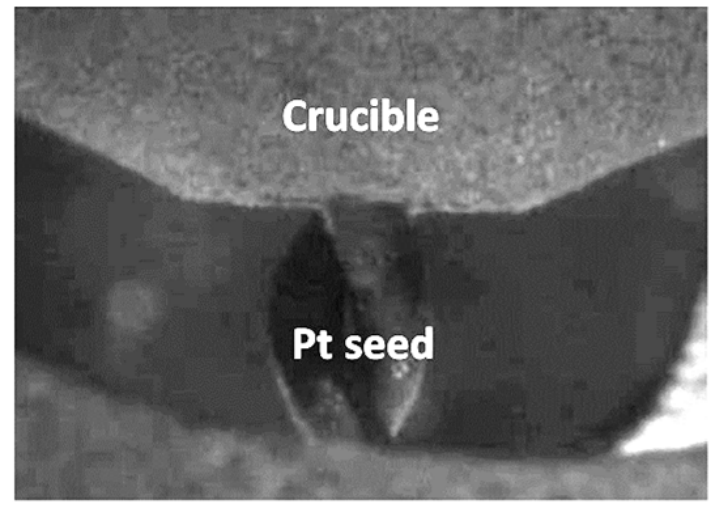

(b)

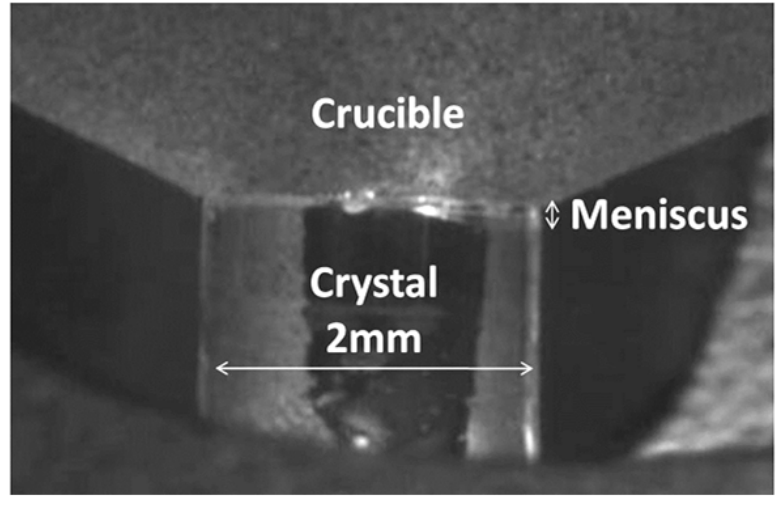

Fig. 1. Liquid-solid interface during crystal growth observed by the CCD camera at (a) initiation of growth with first seed contact to the melt and (b) stable growth state. 
(a)

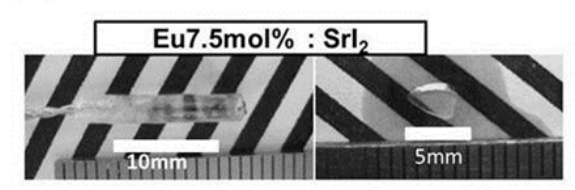

Eu7.5mol\%,Gd1 $1 \mathrm{~mol} \%$ : $\mathrm{Srl}_{2}$

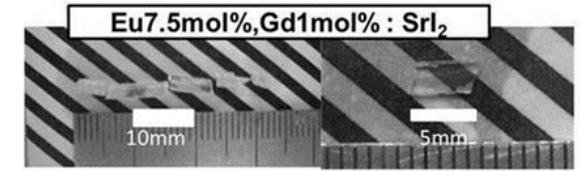

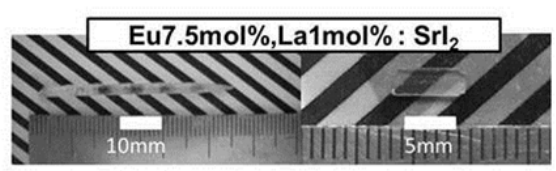

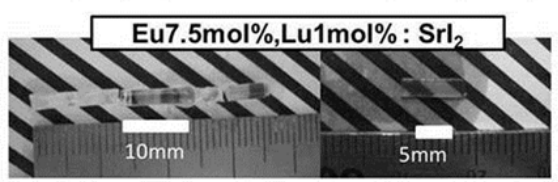

(b)

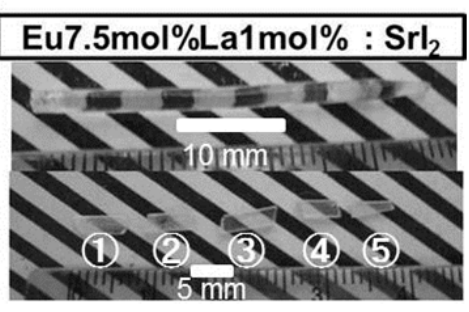

Fig. 2. (a) As-grown and polished Eu:SrI ${ }_{2}$ and La, Ga, or Lu 1mol\% co-doped Eu7.5mol\%: $\mathrm{SrI}_{2}$ crystals. (b) As-grown and polished La $1 \mathrm{~mol} \%$ co-doped Eu 7.5mol\%: $\operatorname{SrI}_{2}$ grown with complete solidification of the melt (100\% solidification fraction). 


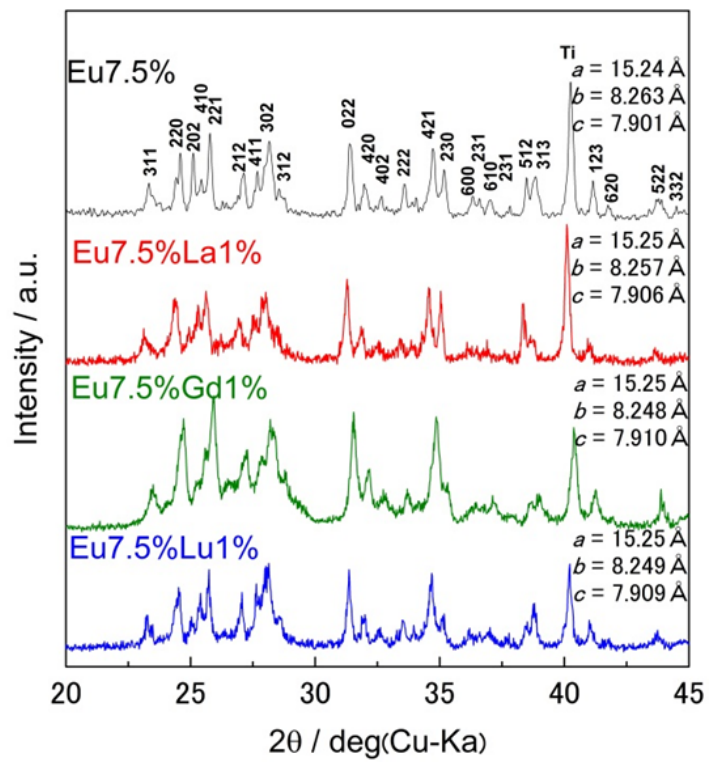

Fig. 3. Powder XRD patterns and lattice parameters of Eu: $\mathrm{SrI}_{2}$ and $\mathrm{La}, \mathrm{Gd}$, or Lu co-doped $\mathrm{Eu}: \mathrm{SrI}_{2}$ crystals. 


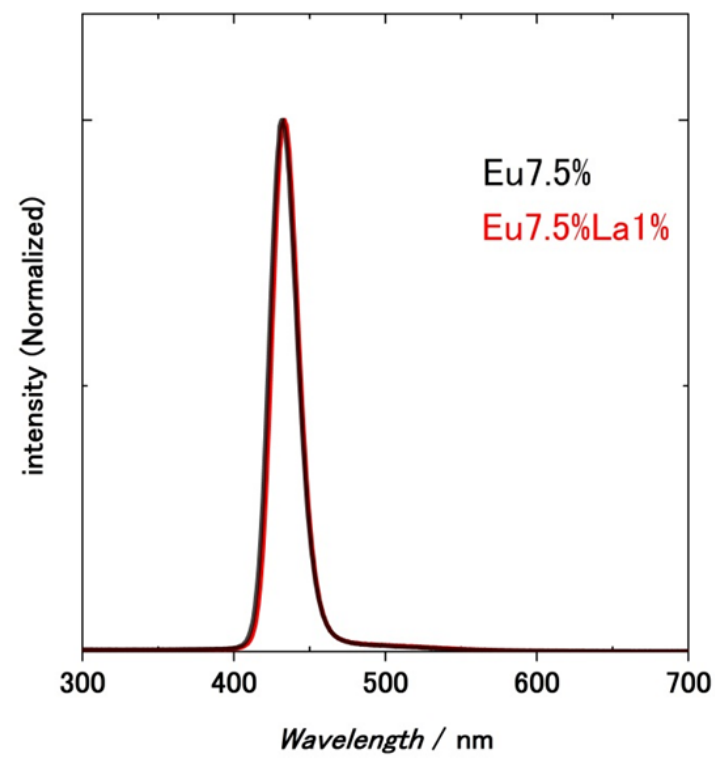

Fig. 4. Radioluminescence spectra of Eu: $\mathrm{SrI}_{2}$ and La co-doped Eu:SrI 2 specimens under X-ray irradiation. 
(a)

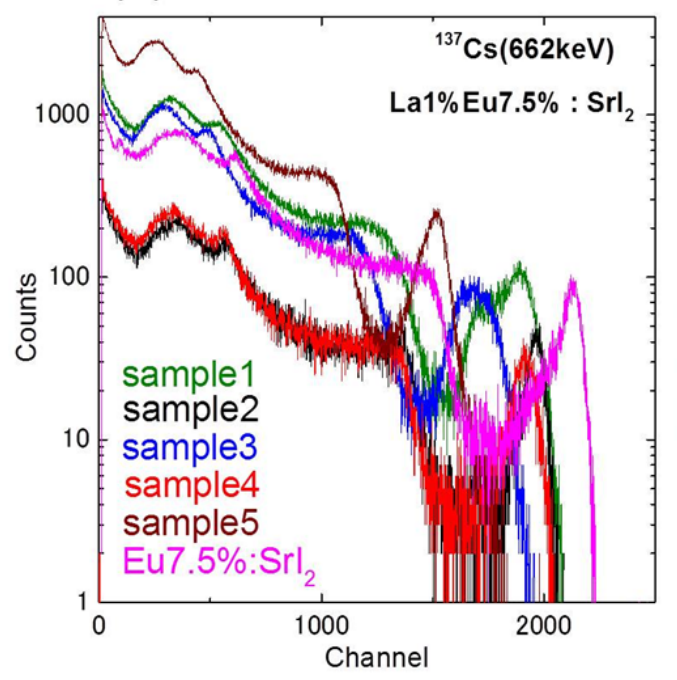

(b)

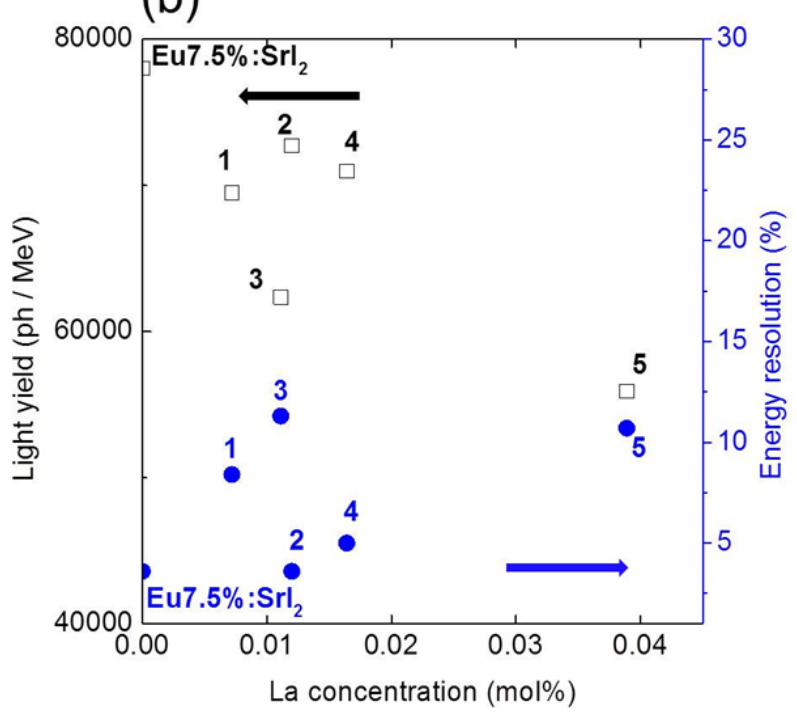

Fig.5. (a) Pulse-height spectra of Eu:SrI $\mathrm{Sn}_{2}$ and La co-doped Eu:SrI $\mathrm{S}_{2}$ specimens and (b) La concentration dependence of light yield and energy resolution. 


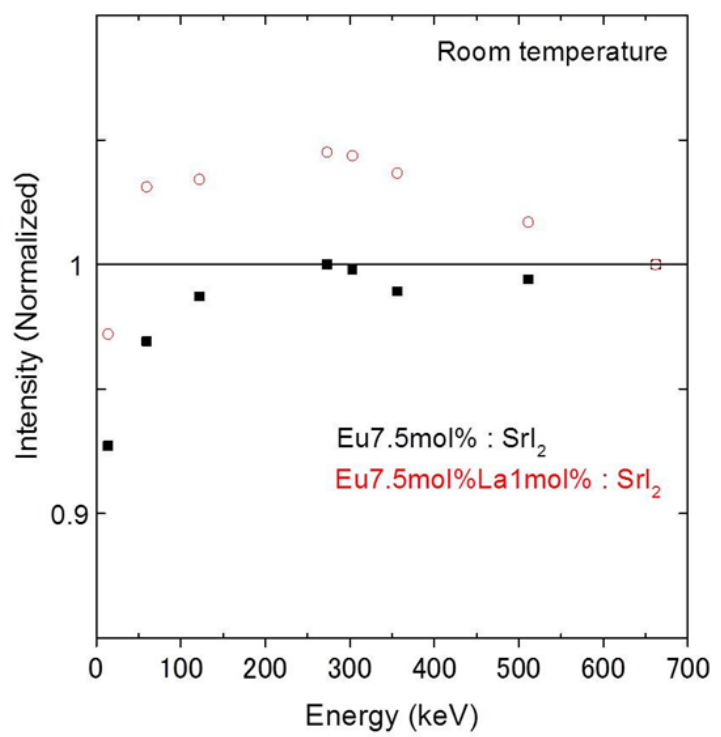

Fig. 6. Non-proportionality of Eu: $\mathrm{SrI}_{2}$ (solid squares) and La co-doped Eu: $\mathrm{SrI}_{2}$ (open circles) specimens under $\gamma$-ray irradiation from ${ }^{137} \mathrm{Cs},{ }^{22} \mathrm{Na},{ }^{57} \mathrm{Co},{ }^{133} \mathrm{Ba}$ and ${ }^{241} \mathrm{Am}$. 


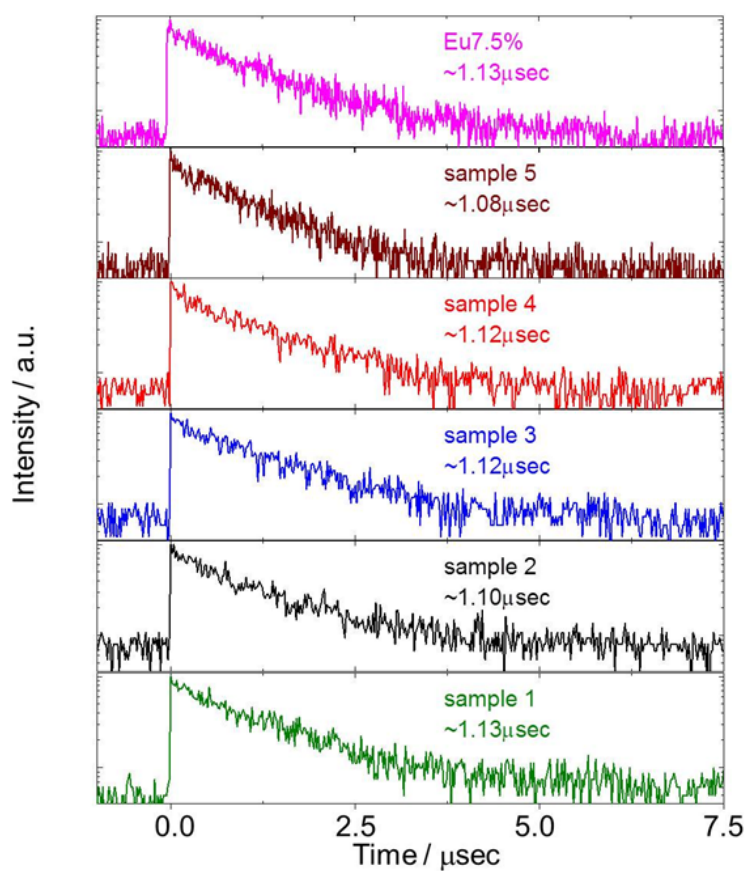

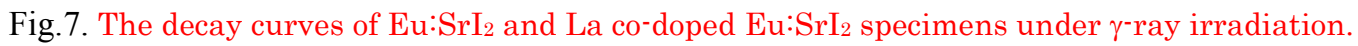

